\title{
Percepções da equipe multiprofissional de um Centro de Atenção Psicossocial sobre as motivações que levam o sujeito ao comportamento suicida
}

\author{
Perceptions of the multiprofessional team of a Center for Psychosocial Care on the \\ motivations that lead the subject with mental disorder to suicidal behavior
}

\section{Percepciones del equipo multiprofesional de un Centro de Atención Psicosocial sobre las motivaciones que llevan al sujeto con trastorno mental al comportamiento suicida}

\begin{abstract}
Mayara Macedo Melo ${ }^{1}$, Bruna dos Reis Nespoli ${ }^{1}$, Rosane da Silva Santana ${ }^{2}$, Francisco Lucas de Lima Fontes ${ }^{1 *}$, José Gilvam Araújo Lima Junior ${ }^{1}$, Francisco Rafael de Carvalho ${ }^{1}$, Andréa Luíza de Oliveira Milanêz ${ }^{3}$, Jackson Menezes Duarte ${ }^{1}$, Taciane Aparecida Dias dos Santos ${ }^{1}$, Joelma da Silva Porto $^{1}$, Mara Monize Pinheiro Mendes ${ }^{4}$, Vanessa Maria Oliveira Viana ${ }^{5}$, Vera Alice Oliveira Viana ${ }^{6}$, Nayara Regina da Silva de Sousa1 ${ }^{1}$, Alan Danilo Teixeira Carvalho¹.
\end{abstract}

\section{RESUMO}

Objetivo: Analisar as percepções da equipe multiprofissional de um Centro de Atenção Psicossocial sobre as motivações que levam o sujeito ao comportamento suicida. Métodos: Trata-se de um estudo descritivo com abordagem qualitativa, desenvolvido em um Centro de Atenção Psicossocial tipo II de Teresina, estado do Piauí. Os sujeitos da pesquisa foram 10 profissionais de saúde sendo quatro enfermeiros, quatro psicólogos e dois médicos psiquiatras. Os dados foram coletados por meio de entrevista semiestruturada entre os meses de fevereiro a maio de 2018, após aprovação do Comitê de Ética em Pesquisa. Resultados: Percebeu-se por meio dos discursos que aspectos relacionados problemas sociais, familiares e amorosos, bullying, utilização de substâncias psicoativas e histórico de transtornos mentais preexistentes são gatilhos para apresentação de comportamentos e ideações suicidas. Conclusão: A identificação precoce de sinais, sintomas e comportamentos de alerta para o suicídio são essenciais para que profissionais, familiares e entes próximos reconheçam possíveis ideações suicidas.

Palavras-chave: Suicídio, Equipe multiprofissional, Saúde Mental.

\begin{abstract}
Objective: To analyze the perceptions of the multiprofessional team of a Psychosocial Care Center about the motivations that lead the subject to suicidal behavior. Methods: This is a descriptive study with a qualitative approach, developed in a Psychosocial Care Center type II of Teresina, state of Piauí. The research subjects were 10 health professionals, four nurses, four psychologists and two psychiatrists. Data were collected through a semistructured interview between February and May 2018, after approval of the Research Ethics Committee. Results: Through the discourses, social, family, and love problems, bullying, use of psychoactive substances and history of preexisting mental disorders were perceived as triggers for the presentation of suicidal behaviors and ideations. Conclusion: Early identification of suicide warning signs, symptoms and behaviors is essential for professionals, families and close relatives to recognize possible suicidal ideation.
\end{abstract}

Keywords: Suicide, Patient care team, Mental health.

\footnotetext{
${ }^{1}$ Faculdade UNINASSAU - Campus Redenção. Teresina, Piauí. *E-mail: lucasfontesenf@hotmail.com

2 Universidade Federal do Ceará. Fortaleza, Ceará.

${ }^{3}$ Centro Universitário UNINOVAFAPI. Teresina, Piauí.

${ }^{4}$ Faculdade FACID - Wyden. Teresina, Piauí.

${ }^{5}$ Universidade Federal do Piauí. Teresina, Piauí.

${ }^{6}$ Centro Universitário Santo Agostinho. Teresina, Piauí.
} 


\section{RESUMEN}

Objetivo: Analizar las percepciones del equipo multiprofesional de un Centro de Atención Psicosocial sobre las motivaciones que llevan al sujeto al comportamiento suicida. Métodos: Se trata de un estudio descriptivo con abordaje cualitativo, desarrollado en un Centro de Atención Psicosocial tipo II de Teresina, estado de Piauí. Los sujetos de la investigación fueron 10 profesionales de salud siendo cuatro enfermeros, cuatro psicólogos y dos médicos psiquiatras. Los datos fueron recolectados por medio de una entrevista semiestructurada entre los meses de febrero a mayo de 2018, tras la aprobación del Comité de Ética en Investigación. Resultados: Se percibió por medio de los discursos que aspectos relacionados problemas sociales, familiares y amorosos, bullying, utilización de sustancias psicoactivas e histórico de trastornos mentales preexistentes son desencadenantes para la presentación de comportamientos e ideaciones suicidas. Conclusión: La identificación temprana de signos, síntomas y comportamientos de alerta para el suicidio son esenciales para que profesionales, familiares y entes cercanos reconozcan posibles ideaciones suicidas.

Palabras-clave: Suicidio, Grupo de atención al paciente, Salud mental.

\section{INTRODUÇÃO}

O suicídio é identificado como uma das três principais causas de óbito no mundo, excedendo um milhão de mortes ao ano (BOTEGA NJ, 2014). Tornou-se um problema de saúde pública dentro e fora do Brasil, e de acordo com Waiselfisz JJ (2014), as taxas deste fenômeno cresce junto aos dois maiores grupos de mortalidade violenta, acidentes de trânsito e homicídios, com estimativas de um suicídio para cada 5,5 homicídios e 4,5 mortes no trânsito.

O suicídio é compreendido como um fenômeno complexo e multicausal que repercute socialmente e tem relevante alcance individual, econômico e social (FERREIRA JÚNIOR A, 2015). Caracteriza-se como um ato com resultado letal, iniciado e realizado pelo sujeito consciente. O comportamento suicida é outro termo utilizado pela literatura para referir-se a um ato sem resultado fatal, no qual o sujeito se auto lesiona e/ou apresenta pensamentos persistentes relacionados ao suicídio (SOUZA ACG, et al, 2015).

Daolio ER e Silva JV (2009) explanam que as motivações que levam o indivíduo a cometer tal ato podem variar desde de um transtorno mental pré-existente, histórico familiar, perda súbita de entes próximos a fatores socioculturais. Santos SA, et al., (2009) destacam que o suicídio, em uma classificação psicopatológica, pode ser causado por grave depressão, transtorno de ansiedade generalizado, agorafobia e estresse póstraumático.

O risco de suicídio aumenta de acordo com o número de tentativas e, também está associado a intervalos de tempo menores entre esses esforços de autoextermínio. Estima-se pelo menos dez tentativas graves para um caso de suicídio (VIDAL CEL, et al, 2013). Essas tentativas, caracterizadas como atos de autoagressão, podem levar ao óbito na ausência de atendimento e acompanhamento profissional adequado (SOUZA VS, et al., 2011).

O indivíduo que tenha ideação e/ou comportamento suicida, provavelmente apresenta sofrimento psíquico intenso e precisa de acompanhamento especializado. No Brasil, com a Reforma Psiquiátrica e a regulamentação da Portaria no 336 do Ministério da Saúde, de 19 de fevereiro de 2002, foi criado um serviço especializado para atender indivíduos com transtornos mentais severos e/ou persistentes, inclusive aqueles com comportamentos suicidas. Tais serviços passaram a ser oferecidas pelo Centro de Atenção Psicossocial (CAPS), responsável por prestar serviço comunitário em saúde mental voltado para a reabilitação do sujeito, a estabilidade psíquica e reinserção social (CORDEIRO LRO, et al, 2012).

$O$ atendimento oferecido pelo CAPS tem projeto terapêutico singular voltado ao propósito de reinserção social do indivíduo com apoio e protagonismo da família e conta com atendimento de equipe multiprofissional composta por profissionais de nível superior (médico, enfermeiro, psicólogo, assistente social, pedagogo e/ou terapeuta ocupacional) e de nível médio (técnico de Enfermagem, técnico educacional, artesão e técnico 
administrativo). Outro serviço oferecido no CAPS é o tratamento psicoterapêutico, em que profissionais técnicos realizam atendimento individual especializado com os pacientes, intercalado com dinâmicas em grupo. Entre as atividades realizadas pela equipe multiprofissional destacam-se a escuta qualificada, as orientações sobre manipulação de medicamentos e o encaminhamento a outros serviços de saúde, quando necessário (MINISTÉRIO DA SAÚDE, 2004).

Diante do exposto, o objetivo deste estudo foi analisar as percepções da equipe multiprofissional de um Centro de Atenção Psicossocial sobre as motivações que levam o sujeito ao comportamento suicida.

\section{MÉTODOS}

Trata-se de um estudo descritivo com abordagem qualitativa desenvolvido em um CAPS tipo II do município de Teresina, capital do Piauí. Esse tipo de CAPS oferece serviço com rotina diária no atendimento ao público adulto em município com abrangência populacional entre 70.000 e 200.000 habitantes e conta com atendimento individual e coletivo, visitas domiciliares, execução de oficinas terapêuticas, atividades comunitárias e suporte familiar. O local foi selecionado por meio de sorteio aleatório simples, dentre todos os sete CAPS existentes no município.

Participaram do estudo dez profissionais de saúde sendo quatro enfermeiros, quatro psicólogos e dois médicos psiquiatras. Esses profissionais faziam parte da equipe multiprofissional que prestava assistência aos pacientes em acompanhamento e tratamento em saúde mental. Foram incluídos na pesquisa apenas os profissionais de saúde que realizavam atendimento individual em consultórios e que trabalhavam no CAPS há mais de um ano. E excluídos aqueles que se encontravam de licença ou férias durante a coleta de dados.

A pesquisa seguiu as recomendações da Resolução no 466 do Conselho Nacional de Saúde, e teve seu início após aprovação do Comitê de Ética em Pesquisa da Faculdade de Ciências e Tecnologia do Maranhão (FACEMA) com $n^{\circ}$ do parecer 2.511.143. Todos os participantes foram esclarecidos sobre o estudo e assinaram o Termo de Consentimento Livre e Esclarecido. A representação do nome dos sujeitos foi expressa pelas letras EM, de equipe multiprofissional, seguidas por números romanos (I, II, III, IV...), conforme ordem cronológica de realização das entrevistas, garantindo sigilo e anonimato dos entrevistados durante todo o processo de pesquisa.

A coleta dos dados ocorreu no período de fevereiro a maio de 2018. Utilizou-se um roteiro semiestruturado composto por duas etapas: a primeira, com dados pessoais e profissionais para caracterizar o perfil do grupo entrevistado, e a segunda, composta por duas questões abertas sobre objetivo do estudo.

Para que nenhuma informação relevante fosse perdida ou esquecida, foi utilizado como recurso de apoio um aparelho MP4 para garantir a autenticidade dos depoimentos representados pelas falas dos entrevistados. Os relatos obtidos foram transcritos na íntegra, e empregou-se a técnica do Discurso do Sujeito Coletivo, que compreende um conjunto de falas individuais, de onde são retiradas as ideias centrais para a construção de um discurso comum que representa o pensamento coletivo (BARDIN L, 2016).

\section{RESULTADOS E DISCUSSÃO}

Participaram da pesquisa dez profissionais da equipe multiprofissional do CAPS, sendo quatro enfermeiros, quatro psicólogos e dois médicos psiquiatras. Todos eram do quadro efetivo de servidores do município e possuíam carga horária laboral de 40 horas semanais. A maioria dos profissionais selecionados eram do gênero feminino (60\%). Quanto a titulação acadêmica, um dos profissionais detinha o título de mestre e os demais possuíam especialização em saúde mental e/ou áreas afins. A média de experiência desses profissionais em saúde mental foi de 5 anos e o desvio padrão foi de 1,15 ano.

Segundo o Boletim de Informação e Saúde do Piauí de 2017, no ano de 2015, o índice de mortalidade por causas externas na faixa etária entre 15 a 29 anos chegou a atingir $72 \%$ do total no Estado. Desses, 8,1\% foram diagnosticados como suicídio. Ainda, entre os anos de 2008 a 2015, o coeficiente de mortalidade por 
suicídio passou de 10,0 e 4,1 para os gêneros masculino e feminino, respectivamente, para 13,2 e 3,7 na comparação com 2015. Tais dados, contudo, não quantificam os números de tentativas, por vezes superiores ao número de esforços de autoextermínio consumados (BOTEGA NJ, 2014).

Para contemplar um cuidado integral à pessoa faz-se necessário um olhar holístico sobre o indivíduo, considerando sua história pregressa no que concerne ao seu meio familiar, social, cultural e histórico rompendo possíveis barreiras que venham a contribuir para a assistência à saúde do paciente que esteja em sofrimento psíquico intenso e tenha atentado contra sua própria vida, com o apoio de uma equipe multiprofissional (GUTIERREZ BAO, 2014). Quando questionados sobre as motivações para 0 comportamento suicida, as percepções de EM I, II, III, IV, V, VI e VII foram diversas, porém semelhantes, evidenciando problemas como desemprego, vínculo familiar estremecido e/ou rompido, estando a maioria dos casos relatados vinculados majoritariamente a uma patologia.

"[...] Os motivos podem ser a doença mental não tratada, desemprego, desilusão amorosa... são esses os que eu já tive contato [...]" - EM I

“[...] Depressão, falta de perspectiva em relação ao futuro, sentimento de não valia, sensação de que sua problemática pessoal não tem solução. Visão do suicídio como a única solução, sofrimento psíquico intenso e desejo de acabar com este sofrimento [...]" EM II

“[...] Primeiro o que a gente mais vê aqui é o transtorno mental, paciente já tem um transtorno mental, já tem uma propensão bem grande, né? Principalmente os que são bipolares. Desses transtornos mentais a frequência maior dessas ideações suicidas, ou ideias ou tentativas mesmo - porque a gente tem muitos casos de tentativas - são o transtorno de bipolaridade e os pacientes depressivos também [...]" - EM III

"[...] Dos que a gente atende aqui a principal motivação da maioria dos pacientes é o episódio de depressão... Também os esquizofrênicos com as vozes de comando, tá entendendo? Essas vozes de comando algumas vezes 'manda' a pessoa cometer o suicídio. $E$ aí muitas vezes aconteceram vários casos aqui que nós recebemos de pacientes que cometeram tentativas por conta das vozes de comando. A maioria é a depressão [...]" - EM IV

"[...] A questão como consequência da depressão, também como fator de fuga, as pessoas... elas não querem enfrentar o problema ou não sabem enfrentar ou não tem paciência... e tem muito disso, já pensa na morte como uma solução de tudo... então a ideação suicida ele é muito consequência de tentativas frustradas de superar problemas, como uma última saída, que a pessoa não consegue enxergar o fim do problema e vai na possibilidade da morte [...]" - EM V

“[...] primeiro são os casos de depressão grave, a maioria dos nossos casos aqui são depressões graves. Tem outras patologias... Tem a bipolaridade, né? Quando está na fase depressiva, o sujeito pode ter esse comportamento suicida... O esquizofrênico com menor frequência mas, o bipolar e o depressivo grave, aquela depressão que é crônica, que não consegue tratar e que ela já vem consequência de muitos problemas de vida que a pessoa enfrentou [...]" - EM VI

“[...] Basicamente são as patologias, né? As pessoas já vem associadas aí com questões mesmo familiares, amorosas, de trabalho... social mesmo, de uma maneira geral. Então ela não é tratada e evolui para um suicídio. A depressão grave é muito forte, aí tem também a esquizofrenia, são os que mais a gente tem aqui, tem também o transtorno bipolar. Geralmente são esses [...]" - EM VII

Identificou-se pelas falas dos depoentes motivações similares para o comportamento suicida, abrangendo o transtorno mental não tratado. Patologias como o transtorno depressivo maior, a esquizofrenia e o transtorno 
bipolar foram as mais recorrentes nos discursos. Relatou-se também fatores familiares e amorosos que, por razões singulares encontravam-se desestruturados, predispondo o sujeito a sentimentos de desvalia. Os discursos congruentes entre os profissionais diferiram-se minimamente quando expostas as vivências particulares de cada um.

A íntima relação entre suicídio e depressão tem sido tema de discussões, levantando-se questionamentos sobre como a depressão age no psíquico do indivíduo que tentou autoextermínio. Estudo de 2009 realizado pela Associação Brasileira de Psiquiatria com amostra de 15.629 pessoas que se suicidaram apontou que, destes, 90\% haviam sido diagnosticadas previamente com algum transtorno mental e $35,8 \%$ sofriam de depressão maior, evidenciando intensa relação dos suicidas com a patologia (ASSUMPÇÃO GLS, et al, 2018).

Assim como a depressão, a esquizofrenia, caracterizada como um transtorno mental que apresenta sintomas como delírios, alucinações e desorganização do pensamento, é apontada como uma das causas mais comuns de suicídio (SILVA AM, et al., 2016).

Conflitos nos âmbitos social e familiar, perda de entes queridos, inexistência de um componente positivo, divergências de pensamentos são apontados pela literatura como etiologias de suicídio. Histórico familiar de tentativas e sucesso de autoextermínio também são evidenciadas como fatores predisponentes a este fenômeno (SILVA RM, et al., 2015).

Ademais, além da associação do quadro clínico de transtornos mentais preexistentes e fatores que podem predispor o sujeito ao ato suicida, também devem ser levados em consideração sinais de alerta clássicos como anedonia (perda de interesse por atividades que antes davam prazer), insônia, descontrole emocional, impulsividade, autodepreciação, isolamento social, descrença no futuro, condutas autolesivas, dificuldades de discernimento, incapacidade de lidar/resolver problemas, agressividade e sentimentos de incompetência e culpa (RAMOS VAB, 2017).

EM VIII fez um discurso diferenciado, pontuou como motivação o bullying. Também associou as tentativas e êxitos de autoextermínio a questões referentes a homossexualidade, muitas vezes relacionada a não aceitação de si e rejeição familiar e social, podendo desencadear gatilhos no ambiente de trabalho, local em que o sujeito por vezes encontra-se vulnerável e com dificuldades de interação social. O ambiente laboral, inclusive, também foi lembrado por EM X.

“[...] Já recebi um outro caso que foi em que o sujeito foi exposto. Um bullying que o próprio patrão colocou nas redes sociais a foto dele como se fosse com o namorado, então ele sofreu muito bullying, muito abalo, então isso levou ele... Então tem esse fator, né? Do trabalho, essa motivação assim, de problemas no trabalho... Ah, muito importante! $O$ fator da homossexualidade, também muitas vezes... a dificuldade de enfrentar isso na família e tem esse comportamento suicida... aparecem bastante também. Os transtornos... os depressivos, né? dentro do tratamento eles tem muito esses comportamentos suicida, e também, os outros também, tem o borderline que apresentam comportamentos. A questão do auto preconceito, a dificuldade de eles aceitarem a doença e de tudo que eles sofrem de preconceito, né? Todas essas questões... estão cansados, esse tratamento muito duradouro, a incompreensão da família [...]" - EM VIII

"[...] Temos recebido muitos pacientes com problemas no trabalho, que vem com dificuldade no trabalho, às vezes de... e precisam se afastar e desenvolvem algum transtorno depressivo e em todo esse contexto eles acabam tendo o comportamento. Alguns pacientes meus vieram mesmo desse ambiente de trabalho, né? Cada caso é um caso... por exemplo, recebi um paciente, por exemplo, com relatos de estresse no atendimento de telemarketing, então tem muita pressão, muito estresse, muito adoecimento... é todo esse contexto [...]" - EMX 
As experiências sociais vivenciadas pela população de lésbicas, gays, bissexuais, transexuais e intersexuais (LGBTI+) são, em sua maioria, de ridicularização, violência e hostilidade, usurpando direitos básicos dessa parcela da sociedade, como a convivência respeitosa, sendo repreendidos por seus comportamentos e posturas, predispondo esses sujeitos a fragilização da saúde mental (BAÉRE F, 2018).

Frequentemente vítimas de homofobia, a violência contra LGBTI+ é realidade em nossa sociedade. Esse fenômeno manifesta-se nas relações por meio de ataques físicos, verbais, psicológicos e sexuais (NATARELLI TRP, et al, 2015). Sob a visão da Psicossociologia, todo o segmento LGBTI+ encara humilhações implícitas, por vezes sutis, o que ocasiona repercussões psicológicas na vida desses sujeitos (SOUZA MCA e RAMBALDI M, 2018).

Também é íntima a relação entre bullying e suicídio, sobretudo em âmbito escolar. Crianças e adolescentes ainda não possuem maturidade totalmente constituída, alguns apresentam comportamentos violentos e conflituosos, atacando pontos frágeis de seus pares, o que propicia um campo ideal para comportamentos e ideações suicidas (SOUSA GS, et al., 2017).

O bullying é classificado como direto e indireto. O direto caracteriza-se por ameaças, ataques físicos, termos pejorativos ao se referir a outro sujeito, furtos ou qualquer ação que gere desconforto na vítima. $O$ indireto, por sua vez, relaciona-se ao isolamento da vítima; nesta modalidade, a aparência física, comportamental ou emocional da vítima fazem com elas sejam "escolhidas". Vulneráveis a essas ofensas, a vítima evolui para transtornos como autoestima prejudicada, comportamentos depressivos, ideações suicidas e violência (LOPES NETO A, 2005).

As situações de mal-estar no ambiente de trabalho e temor ao desemprego são condições que também fragilizam a convivência social e propiciam ao trabalhador sentimentos negativos de tristeza e medo, condições que caracterizam-se como campo fértil para o estabelecimento de patologias que afetam a saúde física e mental da pessoa (VENCO S e BARRETO M, 2014).

O trabalho assume papel social, não significa apenas uma atividade com compensação financeira. Por meio dele os indivíduos podem relacionar-se e construir vínculos ao passo em que também pode fortalecer a função psicológica, pois abrange elementos identitários, sociais e profissionais que favorecem 0 reconhecimento e a elevação da autoestima (FREITAS ME, 2011).

A motivação explicitada pelo depoente EM IX pontua a dependência química como fator que suscita ao comportamento, onde o paciente sob tratamento, em detrimento da abstinência, apresenta episódios depressivos estabelecendo grandes chances de ideações suicidas, possibilitando alcançar êxito em tentativa.

"[...] pacientes que tem dependência química e durante a fase de desintoxicação, durante a abstinência ele experimenta sintomas depressivos que geram uma angústia muito grande e que pode levar ao suicídio. E claro os depressivos de uma forma geral vão tender a ter uma baixa autoestima, uma perda de esperança, e começa a ter pensamentos de morte recorrentes, ideação suicida, planeja e acaba tentando pôr fim na própria vida [...]" - EMIX

A dependência e abuso de substâncias psicoativas configura-se um grave problema de saúde pública, impactando negativamente relações parentais, sociedade e serviços públicos de saúde. Estima-se que existam de 16 a 39 milhões de adictos no mundo e 183 mil deles vão a óbito em razão de agravos, sendo o Brasil representante de $20 \%$ do consumo mundial de cocaína (CANTÃO L e BOTTI NCL, 2016).

O juízo crítico do indivíduo é totalmente alterado após o uso de substâncias psicoativas. Sob efeito de substâncias o sujeito pode adotar comportamentos impulsivos e impensados podendo chegar a automutilação e contribuir para o surgimento de ideações e comportamentos de autoextermínio (GONÇALVES REM, et al, 2015).

Discutir o suicídio foi de extrema relevância por permitir a identificação dos diversos fatores que levam os sujeitos a cometerem suicídio. Percebeu-se que aspectos relacionados problemas sociais, familiares e 
amorosos, bullying, utilização de substâncias psicoativas e histórico de transtornos mentais preexistentes são gatilhos para apresentação de comportamentos e ideações suicidas.

\section{CONCLUSÃO}

Grande parte dos sujeitos que cometem o autoextermínio relatam sua intenção previamente. A identificação precoce de sinais, sintomas e comportamentos de alerta para o suicídio são essenciais para que profissionais, familiares e entes próximos reconheçam possíveis ideações suicidas. O conhecimento sobre as motivações contribui para criação de políticas públicas voltadas para a prevenção e posvenção do suicídio, bem como auxilia na articulação de estratégias e manejo de combate a esse fenômeno.

\section{REFERÊNCIAS}

1. ASSUMPÇÃO GLS et al. Depressão e suicídio: uma correlação. Pretextos, 2018; 3(5): 313-333.

2. BAÉRE F. Análise da produção discursiva de notícias sobre o suicídio de LGBTS em um jornal impresso do Distrito Federal. Revista Ártemis, 2018; 25(1): 74-88.

3. BARDIN L. Análise de conteúdo. 1aㅡ. ed., Edições 70, Brasil, 2016.

4. BOTEGA NJ. Comportamento suicida: epidemiologia. Psicologia USP, 2014; 25(3): 231-236.

5. BRASIL. Ministério da Saúde. Secretaria de Atenção à Saúde. Departamento de Ações Programáticas Estratégicas. Saúde mental no SUS: os centros de atenção psicossocial. Brasília: Ministério da Saúde, 2004.

6. CANTÃO L, BOTTI NCL. Comportamento suicida entre dependentes químicos. Revista Brasileira de Enfermagem, 2016; 69(2): 389-396.

7. CORDEIRO LRO et al. Produção científica sobre os Centros de Atenção Psicossocial. Revista da Escola de Enfermagem da USP, 2012; 26(1): 119-23.

8. DAOLIO ER, SILVA JV. Os significados e os motivos do suicídio: as representações sociais de pessoas residentes em Bragança Paulista. Bioethikos, 2009; 3(1): 68-76.

9. FERREIRA JUNIOR A. O comportamento suicida no Brasil e no mundo. Revista Brasileira de Psicologia, 2015; 2(1): 15-28.

10. FREITAS ME. Suicídio, um problema organizacional. GV Executivo, 2011; 10(1): 54-57.

11. GONÇALVES REM et al. Uso de álcool e suicídio. Saúde, Ética e Justiça, 2015; 20(1): 9-14.

12. GUTIERREZ BAO. Assistência Hospitalar na tentativa de suicídio. Psicologia USP, 2014; 25(3): 262-269.

13. LOPES NETO A. Bullying: comportamento agressivo entre estudantes. Jornal de Pediatria, 2005; 81(5): 164-172.

14. NATARELLI TRP et al. O impacto da homofobia na saúde do adolescente. Escola Anna Nery, 2015; 19(4): 664-670.

15. RAMOS VAB. Como prevenir o suicídio. Psicologia PT, 2017; 10(68): 1-15.

16. SANTOS SA et al. Prevalência de Transtornos Mentais nas Tentativas de Suicídio em um Hospital de Emergência no Rio de Janeiro. Cadernos de Saúde Pública, 2009; 25(9): 2064-2074.

17. SILVA AM et al. Esquizofrenia: uma revisão bibliográfica. Revista UNILUS Ensino e Pes quisa, 2016; 13(30): 18-25.

18. SILVA RM et al. Influências dos problemas e conflitos familiares nas ideações e tentativas de suicídio de pessoas idosas. Ciência \& Saúde Coletiva, 2015; 20(6): 1703-1710.

19. SOUSA GS. Revisão de literatura sobre o suicídio na infância. Ciência \& Saúde Coletiva, 2017; 22(9): 3099-3110.

20. SOUZA ACG et al. Suicídio na Adolescência: Revisão de Literatura. Revista Uningá, 2015; 43(1):95-98.

21. SOUZA MCA, RAMBALDI M. "Moonlight - sob a luz do luar" e o sofrimento emocional de homossexuais. Revista Trabalho Encena, 2018; 3(3): 172-186.

22. SOUZA VS et al. Tentativas de suicídio e mortalidade por suicídio em um município no interior da Bahia. Jornal Brasileiro de Psiquiatria, 2011; 60(4): 294-300.

23. VENCO S, BARRETO M. O sentido social do suicídio no trabalho. Revista do Tribunal Superior do Trabalho, 2014; 80(1): 294-302.

24. VIDAL CEL et al. Tentativas de suicídio: fatores prognósticos e estimativa do excesso de mortalidade. Cadernos de Saúde Pública. 2013; 29(1): 175-187.

25. WAISELFISZ JJ. Mapa da Violência no Brasil. Rio de Janeiro: Flacso, 2014. 